\title{
ON THE MONITORING ERROR OF THE SUPREMUM OF A NORMAL JUMP DIFFUSION PROCESS
}

\author{
$\mathrm{AO} \mathrm{CHEN},{ }^{* * *}$ \\ LIMING FENG ${ }^{* * *}$ AND \\ RENMING SONG, ${ }^{* * * * *}$ University of Illinois at Urbana-Champaign
}

\begin{abstract}
We derive an expansion for the (expected) difference between the continuously monitored supremum and evenly monitored discrete maximum over a finite time horizon of a jump diffusion process with independent and identically distributed normal jump sizes. The monitoring error is of the form $a_{0} / N^{1 / 2}+a_{1} / N^{3 / 2}+\cdots+b_{1} / N+b_{2} / N^{2}+b_{4} / N^{4}+\cdots$, where $N$ is the number of monitoring intervals. We obtain explicit expressions for the coefficients $\left\{a_{0}, a_{1}, \ldots, b_{1}, b_{2}, \ldots\right\}$. In particular, $a_{0}$ is proportional to the value of the Riemann zeta function at $\frac{1}{2}$, a well-known fact that has been observed for Brownian motion in applied probability and mathematical finance.
\end{abstract}

Keywords: Normal jump diffusion process; supremum; discrete monitoring; Spitzer's identity; Euler-Maclaurin formula; Riemann zeta function; Lerch transcendent

2010 Mathematics Subject Classification: Primary 60G51; 60J75; 91G60

Secondary $65 \mathrm{C} 20$

\section{Introduction and main results}

Jump diffusions and pure-jump Lévy processes are often used to model the dynamics of asset prices and other financial variables in mathematical finance [4], [7], [23]. They naturally incorporate extreme price movements due to jumps that are difficult to capture by Brownian motion which is underlying the celebrated Black-Scholes-Merton model [3], [20]. The extremum of such a process is of interest when derivatives with barrier and lookback features are evaluated [10], [11], [15], [16], [17], [18], [19], [22].

In this paper, we consider the monitoring error for the supremum of Merton's normal jump diffusion process $[21]$ defined on a complete filtered probability space $(\Omega, \mathcal{F}, \mathbb{F}, \mathrm{P})$ with the filtration $\mathbb{F}=\left\{\mathcal{F}_{t}, t \geq 0\right\}$ satisfying the usual conditions. The process takes the form

$$
X_{t}=\mu t+\sigma B_{t}+\sum_{i=1}^{N_{t}} Z_{i},
$$

where $\mu \in \mathbb{R}, \sigma>0, B_{t}$ is a standard Brownian motion, $N_{t}$ is a Poisson process with intensity $\lambda>0$, and $\left\{Z_{i}, i \geq 1\right\}$ are independent and identically distributed (i.i.d.) jump sizes with

\footnotetext{
Received 25 January 2011; revision received 26 June 2011.

* Postal address: Department of Mathematics, University of Illinois at Urbana-Champaign, Urbana, IL 61801, USA.

** Email address: aochen2@illinois.edu

*** Postal address: Department of Industrial and Enterprise Systems Engineering, University of Illinois at UrbanaChampaign, Urbana, IL 61801, USA. Email address: fenglm@illinois.edu

**** Email address: rsong@illinois.edu

Research partially supported by the National Science Foundation, under grants CMMI-0927367 and CMMI-1029846.
} 
a normal distribution $N\left(\tilde{m}, s^{2}\right)$ for some $\tilde{m} \in \mathbb{R}$ and $s>0$. The Brownian motion, the Poisson process, and the jump sizes are independent. The limiting case $\lambda=0$ is simply a drifted Brownian motion. Much is known about such a process. For example, its characteristic function admits a very simple form. Moreover, the transition probability density of $X$ is also known and given by (see [7])

$$
p(t, x)=\mathrm{e}^{-\lambda t} \sum_{k=0}^{\infty} \frac{(\lambda t)^{k}}{k !} \frac{1}{\sqrt{2 \pi\left(\sigma^{2} t+k s^{2}\right)}} \exp \left(-\frac{(x-\mu t-k \tilde{m})^{2}}{2\left(\sigma^{2} t+k s^{2}\right)}\right) .
$$

We consider the supremum of $X$ on a fixed time horizon $[0, T]$. Without loss of generality, we take $T=1$. We are interested in characterizing the (expected) monitoring error for the supremum when the process is monitored discretely with a constant monitoring interval $T / N$ :

$$
\mathrm{E}\left[\sup _{0 \leq t \leq 1} X_{t}-\max _{0 \leq n \leq N} X_{n / N}\right]
$$

The above quantity appears in various application areas and has been well studied in the case of a drifted Brownian motion $X_{t}=\mu t+\sigma B_{t}$. In [2], it was shown that (2) is of the form $-\sigma \zeta\left(\frac{1}{2}\right) / \sqrt{2 \pi N}+O(1 / N)$, where $\zeta(s)$ is the Riemann zeta function. This result is used to establish that the order of strong convergence when a reflected Brownian motion is simulated using the Euler discretization is $\frac{1}{2}$. The same result is also derived in [6], although in a different form, when two passive algorithms for global optimization of continuous functions on onedimensional domains are studied. The coefficient for $1 / N$ is further derived in [5] when correction formulae are designed for pricing discretely monitored lookback options using continuous lookback option pricing formulae in the Black-Scholes-Merton model. More recently, the above results have been greatly sharpened in [14], where a complete expansion of (2) in terms of $1 / N$ was obtained. More specifically, let $\mathcal{N}(x)$ be the standard normal cumulative distribution function, and let $g(t)=\mu \mathcal{N}(\mu \sqrt{t})+\mathrm{e}^{-\mu^{2} t / 2} / \sqrt{2 \pi t}$. Let $g^{(i)}, i \geq 1$, be the $i$ th derivative of $g$, and let $\left\{B_{k}, k \geq 0\right\}$ be the Bernoulli numbers (see [12])

$$
B_{k}=\sum_{i=0}^{k} \sum_{j=0}^{i}(-1)^{j}\left(\begin{array}{l}
i \\
j
\end{array}\right) \frac{j^{k}}{i+1}, \quad k \geq 0 .
$$

For all odd $k>1, B_{k}=0$, and the first five values of the Bernoulli numbers are $B_{0}=1$, $B_{1}=-\frac{1}{2}, B_{2}=\frac{1}{6}, B_{4}=-\frac{1}{30}$, and $B_{6}=\frac{1}{42}$. Then, for a drifted Brownian motion, (2) admits the following expansion.

Theorem 1. ([14].) Let $X_{t}=\mu t+B_{t}$, where $\mu \in \mathbb{R}$ and $B_{t}$ is a standard Brownian motion. Then, for any integer $m \geq 1$, we have

$$
\begin{aligned}
\mathrm{E}\left[\sup _{0 \leq t \leq 1} X_{t}-\max _{0 \leq n \leq N} X_{n / N}\right]= & -\frac{\zeta(1 / 2)}{\sqrt{2 \pi N}}-\frac{2 g(1)-\mu}{4 N}-\sum_{i=1}^{m} \frac{B_{2 i}}{(2 i) !} \frac{g^{(2 i-1)}(1)}{N^{2 i}} \\
& -\frac{1}{\sqrt{2 \pi N}} \sum_{n=0}^{\infty} \frac{\zeta(-1 / 2-n)(-1 / 2)^{n}}{n !(2 n+1)(2 n+2)}\left(\frac{\mu}{\sqrt{N}}\right)^{2 n+2} \\
& +O\left(\frac{1}{N^{2 m+2}}\right)
\end{aligned}
$$


In this paper, we follow the approach of [14] and extend the above result to Merton's normal jump diffusion process. Before we state the main result of this paper, we introduce some notation. Throughout this paper, we will use $\operatorname{Erf}(x)$ and $\operatorname{Erfc}(x)$ to denote the error function and the complementary error function:

$$
\operatorname{Erf}(x)=\frac{2}{\sqrt{\pi}} \int_{0}^{x} \mathrm{e}^{-t^{2}} \mathrm{~d} t=2 \mathcal{N}(\sqrt{2} x)-1
$$

and

$$
\operatorname{Erfc}(x)=1-\operatorname{Erf}(x)=\frac{2}{\sqrt{\pi}} \int_{x}^{\infty} \mathrm{e}^{-t^{2}} \mathrm{~d} t=2 \mathcal{N}(-\sqrt{2} x) .
$$

In the remainder of this paper, we set $a=\left(2 \lambda \sigma^{2}+\mu^{2}\right) /\left(2 \sigma^{2}\right)$ and $a(x)=\left(2 \lambda \sigma^{2}+x^{2}\right) /\left(2 \sigma^{2}\right)$, and define

$$
\begin{aligned}
f_{1}(t) & =\mathrm{e}^{-\lambda t} \operatorname{Erfc}\left(-\frac{\mu}{\sqrt{2 \sigma^{2}}} \sqrt{t}\right), \\
f_{2}(t) & =\frac{1}{\sqrt{t}} \mathrm{e}^{-a t}, \\
g(t, x) & =\sqrt{t} \mathrm{e}^{-a(x) t}, \\
p_{k}(t) & =\mathrm{e}^{-\lambda t} t^{k-1} \operatorname{Erfc}\left(-\frac{\mu t+k \tilde{m}}{\sqrt{2\left(\sigma^{2} t+k s^{2}\right)}}\right), \quad k \geq 1, \\
q_{k}(t) & =t p_{k}(t), \quad k \geq 1, \\
r_{k}(t) & =\mathrm{e}^{-\lambda t} t^{k-1} \sqrt{\sigma^{2} t+k s^{2}} \exp \left(-\frac{(\mu t+k \tilde{m})^{2}}{2\left(\sigma^{2} t+k s^{2}\right)}\right), \quad k \geq 1 .
\end{aligned}
$$

For a function $f(t)$ and $i \geq 1, f^{(i)}(t)$ denotes the $i$ th derivative of $f(t)$. Moreover, $g^{(i)}(t, x)$ denotes the $i$ th derivative of $g(t, x)$ with respect to $t$. The main result of this paper is as follows.

Theorem 2. Let $X_{t}=\mu t+\sigma B_{t}+\sum_{i=1}^{N_{t}} Z_{i}$, where $\mu \in \mathbb{R}, \sigma>0$, $B_{t}$ is a standard Brownian motion, $N_{t}$ is a Poisson process with intensity $\lambda>0, Z_{i} \sim N\left(\tilde{m}, s^{2}\right)$ are i.i.d. with $\tilde{m} \in \mathbb{R}$ and $s>0$, and $B_{t}, N_{t}, Z_{i}, i \geq 1$, are independent. Then, for anyinteger $N>\left(2 \lambda \sigma^{2}+\mu^{2}\right) /\left(4 \pi \sigma^{2}\right)$ and $m \geq 1$,

$$
\mathrm{E}\left[\sup _{0 \leq t \leq 1} X_{t}-\max _{0 \leq n \leq N} X_{n / N}\right]=-\frac{\sigma \zeta(1 / 2)}{\sqrt{2 \pi N}}+\frac{b_{1}}{N}+\sum_{n=1}^{\infty} \frac{a_{n}}{N^{n+1 / 2}}+\sum_{i=1}^{m} \frac{b_{2 i}}{N^{2 i}}+O\left(\frac{1}{N^{2 m+2}}\right) .
$$

The coefficients are given by

$$
a_{n}=-\frac{\zeta(1 / 2-n)}{n ! \sqrt{2 \pi}}\left(\sigma\left(-\lambda-\frac{\mu^{2}}{2 \sigma^{2}}\right)^{n}+\frac{n \mu}{\sigma} \int_{0}^{\mu}\left(-\lambda-\frac{x^{2}}{2 \sigma^{2}}\right)^{n-1} \mathrm{~d} x\right), \quad n \geq 1,
$$

where $\zeta(s)$ is the Riemann zeta function, $\zeta\left(\frac{1}{2}\right) \approx-1.46035451$,

$$
\begin{array}{r}
b_{1}=\frac{1}{4}\left(\mu-\mu \mathrm{e}^{-\lambda} \operatorname{ErfC}\left(-\frac{\mu}{\sqrt{2 \sigma^{2}}}\right)+\tilde{m} \lambda \operatorname{ErfC}\left(-\frac{\tilde{m}}{\sqrt{2 s^{2}}}\right)\right. \\
\left.-\sum_{k=1}^{\infty} \frac{\lambda^{k}}{k !}(\mu+\tilde{m} k) \mathrm{e}^{-\lambda} \operatorname{ErfC}\left(-\frac{\mu+k \tilde{m}}{\sqrt{2\left(\sigma^{2}+k s^{2}\right)}}\right)\right)
\end{array}
$$




$$
\begin{aligned}
+\frac{1}{2 \sqrt{2 \pi}}\left(\lambda s \exp \left(-\frac{\tilde{m}^{2}}{2 s^{2}}\right)-\sigma \exp \left(-\left(\lambda+\frac{\mu^{2}}{2 \sigma^{2}}\right)\right)\right. \\
\left.-\sum_{k=1}^{\infty} \frac{\lambda^{k}}{k !} \mathrm{e}^{-\lambda} \sqrt{\sigma^{2}+k s^{2}} \exp \left(-\frac{(\mu+k \tilde{m})^{2}}{2\left(\sigma^{2}+k s^{2}\right)}\right)\right)
\end{aligned}
$$

and, for $i \geq 1$,

$$
\begin{aligned}
b_{2 i}=-\frac{B_{2 i}}{(2 i) !}( & \frac{\mu}{2} \lambda^{2 i-1}\left(1-\mathrm{e}^{-\lambda}\right)+\frac{\mu}{\sqrt{2 \pi \sigma^{2}}} \int_{0}^{\mu} g^{(2 i-1)}(1, x) \mathrm{d} x \\
& +\frac{1}{\sqrt{2 \pi}} \sum_{k=1}^{\infty} \frac{\lambda^{k}}{k !}\left(r_{k}^{(2 i-1)}(1)-r_{k}^{(2 i-1)}(0)\right)+\frac{\sigma}{\sqrt{2 \pi}} f_{2}^{(2 i-1)}(1) \\
& \left.+\frac{1}{2} \sum_{k=1}^{\infty} \frac{\lambda^{k}}{k !}\left(\mu\left(q_{k}^{(2 i-1)}(1)-q_{k}^{(2 i-1)}(0)\right)+\tilde{m} k\left(p_{k}^{(2 i-1)}(1)-p_{k}^{(2 i-1)}(0)\right)\right)\right)
\end{aligned}
$$

Two remarks are in order. First, it is trivial to verify that Theorem 2 reduces to Theorem 1 when $\lambda=0$ and $\sigma=1$. Second, the first term in the expansion for the jump diffusion process is the same as the first term in the expansion for the corresponding drifted Brownian motion without jumps. That is, it does not depend on jumps. Intuitively, due to the nature of the compound Poisson process component in $X_{t}$, the probability that an interval of length $1 / N$ contains one or more jumps is of order $O(1 / N)$. When $N$ increases, the monitoring error due to the drifted Brownian motion component, which is of the order $O(1 / \sqrt{N})$, dominates.

Before we proceed to the proof, we first review some results that will be used later. It is known that the error function admits the following Taylor expansion (see Section 7.1.5 of [1]):

$$
\operatorname{Erf}(x)=\frac{2}{\sqrt{\pi}} \sum_{n=0}^{\infty} \frac{(-1)^{n} x^{2 n+1}}{n !(2 n+1)}=\frac{2}{\sqrt{\pi}}\left(x-\frac{x^{3}}{3}+\frac{x^{5}}{10}-\cdots\right) \quad \text { for all } x \in \mathbb{R} .
$$

Denote the probability density function of the standard normal distribution by $\phi(x)=$ $(1 / \sqrt{2 \pi}) \exp \left(-\frac{1}{2} x^{2}\right)$. Using $\phi^{(1)}(x)=-x \phi(x)$, it is easy to verify that

$$
\operatorname{ErfC}^{(1)}(x)=-2 \sqrt{2} \phi(-\sqrt{2} x), \quad \operatorname{ErfC}^{(2)}(x)=4 \sqrt{2} x \phi(-\sqrt{2} x) .
$$

Since the normal jump diffusion process is a Lévy process, it corresponds to a random walk when monitored discretely and evenly. One of the important tools that is often used to study the maximum of a random walk is Spitzer's identity [24], which gives an explicit expression for the maximum of a random walk. More specifically, for a random walk $S_{0}=0, S_{n}=$ $\sum_{k=1}^{n} X_{k}, n \geq 1$, with i.i.d. step sizes $\left\{X_{k}, k \geq 1\right\}$, it holds that

$$
\mathrm{E}\left[\max _{0 \leq k \leq n} S_{k}\right]=\sum_{k=1}^{n} \frac{1}{k} \mathrm{E}\left[S_{k}^{+}\right]
$$

where $x^{+}=\max (0, x)$. In our case, we have a discretely monitored normal jump diffusion process $X_{t}$ with monitoring interval $1 / N$ and time horizon $[0,1]$. From the independent and stationary increment properties and Spitzer's identity, we have

$$
\mathrm{E}\left[\max _{0 \leq n \leq N} X_{n / N}\right]=\sum_{n=1}^{N} \frac{1}{n} \mathrm{E}\left[X_{n / N}^{+}\right]=\sum_{n=1}^{N} \frac{1}{n} \int_{0}^{\infty} x p\left(\frac{n}{N}, x\right) \mathrm{d} x .
$$


By the dominated convergence theorem, the expected supremum of $X$ on $[0,1]$ is then given by

$$
\mathrm{E}\left[\sup _{0 \leq t \leq 1} X_{t}\right]=\int_{0}^{1} \frac{1}{t} \int_{0}^{\infty} x p(t, x) \mathrm{d} x \mathrm{~d} t .
$$

We note that the right-hand side of (5) is very close to the trapezoidal sum approximation of the integral on the right-hand side of (6). Expansion of the error of the trapezoidal sum approximation to an integral can thus be used to study the difference between the continuous supremum and discrete maximum. This motivates the use of the Euler-Maclaurin formula, which describes the discretization error of the trapezoidal approximation to an integral (see [8, Theorem 3.4.10, Chapter 3]): if $f \in C^{2 m+2}([a, b]), b-a=N h$ for a positive integer $N \geq 1$, and $h>0$, then

$$
\begin{array}{rl}
\int_{a}^{b} & f(x) \mathrm{d} x-h \sum_{n=1}^{N} f(a+n h) \\
& =-\frac{h}{2}(f(b)-f(a))-\sum_{i=1}^{m} \frac{B_{2 i}}{(2 i) !} h^{2 i}\left(f^{(2 i-1)}(b)-f^{(2 i-1)}(a)\right)-R_{2 m+2},
\end{array}
$$

where

$$
R_{2 m+2}=h^{2 m+2} \int_{a}^{b}\left(B_{2 m+2}-\hat{B}_{2 m+2}\left(\frac{x-a}{h}\right)\right) \frac{f^{(2 m+2)}(x)}{(2 m+2) !} \mathrm{d} x .
$$

Here

$$
B_{i}(x)=\sum_{j=0}^{i}\left(\begin{array}{l}
i \\
j
\end{array}\right) B_{j} x^{i-j}
$$

is the $i$ th Bernoulli polynomial, $\hat{B}_{i}(x)=B_{i}(x-\lfloor x\rfloor)$, and $\lfloor x\rfloor$ is the largest integer that is less than or equal to $x$. Note that $\hat{B}_{i}(x)$ is the periodically extended version of $B_{i}(x)$ on $[0,1]$ and is hence bounded. If $f \in C^{2 m+2}([a,+\infty)), \lim _{b \rightarrow+\infty} f^{(i)}(b)=0,0 \leq i \leq 2 m+1$, and $\int_{a}^{+\infty}\left|f^{(2 m+2)}(x)\right| \mathrm{d} x<\infty$, then the above still holds by replacing $f(b)$ and $f^{(2 i-1)}(b), 1 \leq$ $i \leq m$, by $0, N$ by $+\infty$, and the upper limit $b$ of the integral in $R_{2 m+2}$ by $+\infty$.

For certain integrals that we need to handle, instead of the Euler-Maclaurin formula, wellestablished results in analytic number theory can be applied directly. The following result regarding the Lerch transcendent has been successfully applied in [13] and [14] for studying the maximum of Gaussian random walks and to obtain Theorem 1. The same technique will be used to derive Theorem 2 in this paper. The Lerch transcendent is the analytic continuation of the following infinite series:

$$
\Phi(z, s, v)=\sum_{n=0}^{\infty}(n+v)^{-s} z^{n} .
$$

It has the following expansion for $|\log (z)|<2 \pi, s \neq 1,2,3, \ldots, v \neq 0,-1,-2, \ldots$ (see $[9$, Chapter 1]):

$$
\Phi(z, s, v)=\frac{\Gamma(1-s)}{z^{v}}\left(\log \left(\frac{1}{z}\right)\right)^{s-1}+\frac{1}{z^{v}} \sum_{n=0}^{\infty} \zeta(s-n, v) \frac{(\log (z))^{n}}{n !},
$$

where $\Gamma(x)$ is the gamma function and $\zeta(s, v)=\Phi(1, s, v)$ is the Hurwitz zeta function. In particular, $\zeta(s, 1)$ is the Riemann zeta function $\zeta(s)$. 


\section{Proof of Theorem 2}

Recall that

$$
\begin{gathered}
\mathrm{E}\left[\sup _{0 \leq t \leq 1} X_{t}\right]=\int_{0}^{1} \frac{1}{t} \int_{0}^{\infty} x p(t, x) \mathrm{d} x \mathrm{~d} t, \\
\mathrm{E}\left[\max _{0 \leq n \leq N} X_{n / N}\right]=\sum_{n=1}^{N} \frac{1}{n} \int_{0}^{\infty} x p\left(\frac{n}{N}, x\right) \mathrm{d} x .
\end{gathered}
$$

With the transition density $p(t, x)$ given in (1), we have, by Fubini's theorem,

$$
\begin{aligned}
& \int_{0}^{\infty} x p(t, x) \mathrm{d} x= \mathrm{e}^{-\lambda t} \sum_{k=0}^{\infty} \frac{(\lambda t)^{k}}{k !} \frac{1}{\sqrt{2 \pi\left(\sigma^{2} t+k s^{2}\right)}} \int_{0}^{\infty} x \exp \left(-\frac{(x-\mu t-k \tilde{m})^{2}}{2\left(\sigma^{2} t+k s^{2}\right)}\right) \mathrm{d} x \\
&=\mathrm{e}^{-\lambda t} \sum_{k=0}^{\infty} \frac{(\lambda t)^{k}}{k !}\left(\frac{\mu t+k \tilde{m}}{2} \operatorname{ErfC}\left(-\frac{\mu t+k \tilde{m}}{\sqrt{2\left(\sigma^{2} t+k s^{2}\right)}}\right)\right. \\
&\left.+\sqrt{\frac{\sigma^{2} t+k s^{2}}{2 \pi}} \exp \left(-\frac{(\mu t+k \tilde{m})^{2}}{2\left(\sigma^{2} t+k s^{2}\right)}\right)\right) .
\end{aligned}
$$

Applying Fubini's theorem again, we find that $\mathrm{E}\left[\sup _{0 \leq t \leq 1} X_{t}\right]$ is equal to

$$
\begin{aligned}
\sum_{k=0}^{\infty} \frac{\lambda^{k}}{k !} \int_{0}^{1} \mathrm{e}^{-\lambda t} t^{k-1}( & \frac{\mu t+k \tilde{m}}{2} \operatorname{Erfc}\left(-\frac{\mu t+k \tilde{m}}{\sqrt{2\left(\sigma^{2} t+k s^{2}\right)}}\right) \\
& \left.+\sqrt{\frac{\sigma^{2} t+k s^{2}}{2 \pi}} \exp \left(-\frac{(\mu t+k \tilde{m})^{2}}{2\left(\sigma^{2} t+k s^{2}\right)}\right)\right) \mathrm{d} t .
\end{aligned}
$$

The term corresponding to $k=0$ reduces to

$$
\frac{\mu}{2} \int_{0}^{1} \mathrm{e}^{-\lambda t} \operatorname{Erfc}\left(-\frac{\mu}{\sqrt{2 \sigma^{2}}} \sqrt{t}\right) \mathrm{d} t+\frac{\sigma}{\sqrt{2 \pi}} \int_{0}^{1} \frac{1}{\sqrt{t}} \exp \left(-\left(\lambda+\frac{\mu^{2}}{2 \sigma^{2}}\right) t\right) \mathrm{d} t .
$$

Using the expression above for $\int_{0}^{\infty} x p(t, x) \mathrm{d} x$, with $t$ replaced by $n / N$, we find that $\mathrm{E}\left[\max _{0 \leq n \leq N} X_{n / N}\right]$ is equal to

$$
\begin{aligned}
\sum_{k=0}^{\infty} \frac{\lambda^{k}}{k !} \frac{1}{N} \sum_{n=1}^{N} \mathrm{e}^{-\lambda n / N}\left(\frac{n}{N}\right)^{k-1}( & \frac{\mu n / N+k \tilde{m}}{2} \operatorname{Erfc}\left(-\frac{\mu n / N+k \tilde{m}}{\sqrt{2\left(\sigma^{2} n / N+k s^{2}\right)}}\right) \\
& \left.+\sqrt{\frac{\sigma^{2} n / N+k s^{2}}{2 \pi}} \exp \left(-\frac{(\mu n / N+k \tilde{m})^{2}}{2\left(\sigma^{2} n / N+k s^{2}\right)}\right)\right) .
\end{aligned}
$$

Again, the term corresponding to $k=0$ reduces to

$$
\frac{\mu}{2} \frac{1}{N} \sum_{n=1}^{N} \mathrm{e}^{-\lambda n / N} \operatorname{Erfc}\left(-\frac{\mu}{\sqrt{2 \sigma^{2}}} \sqrt{\frac{n}{N}}\right)+\frac{\sigma}{\sqrt{2 \pi}} \frac{1}{N} \sum_{n=1}^{N} \frac{1}{\sqrt{n / N}} \exp \left(-\left(\lambda+\frac{\mu^{2}}{2 \sigma^{2}}\right) \frac{n}{N}\right) .
$$

To study the monitoring error (2), it suffices to study the differences of the corresponding terms in (8)-(11). More specifically, we have the following representation for (2):

$$
\mathrm{E}\left[\sup _{0 \leq t \leq 1} X_{t}-\sup _{0 \leq n \leq N} X_{n / N}\right]=\frac{\mu}{2} E_{1}+\frac{\sigma}{\sqrt{2 \pi}} E_{2}+\frac{1}{2} E_{3}+\frac{1}{\sqrt{2 \pi}} E_{4} .
$$


Here $E_{1}, E_{2}, E_{3}$, and $E_{4}$ are given by

$$
\begin{gathered}
E_{1}=\int_{0}^{1} f_{1}(t) \mathrm{d} t-\frac{1}{N} \sum_{n=1}^{N} f_{1}\left(\frac{n}{N}\right), \\
E_{2}=\int_{0}^{1} f_{2}(t) \mathrm{d} t-\frac{1}{N} \sum_{n=1}^{N} f_{2}\left(\frac{n}{N}\right), \\
E_{3}=\sum_{k=1}^{\infty} \frac{\lambda^{k}}{k !}\left(\int_{0}^{1}\left(\mu q_{k}(t)+k \tilde{m} p_{k}(t)\right) \mathrm{d} t-\frac{1}{N} \sum_{n=1}^{N}\left(\mu q_{k}\left(\frac{n}{N}\right)+k \tilde{m} p_{k}\left(\frac{n}{N}\right)\right)\right), \\
E_{4}=\sum_{k=1}^{\infty} \frac{\lambda^{k}}{k !}\left(\int_{0}^{1} r_{k}(t) \mathrm{d} t-\frac{1}{N} \sum_{n=1}^{N} r_{k}\left(\frac{n}{N}\right)\right),
\end{gathered}
$$

where $f_{1}, f_{2}, p_{k}, q_{k}$, and $r_{k}$ are defined in (3). For $E_{3}$ and $E_{4}$, the integrands are smooth on $[0,1]$ and the Euler-Maclaurin formula can be used directly. For $E_{1}$ and $E_{2}$, we utilize expansion (7) for the Lerch transcendent.

\subsection{Analysis for $E_{1}$}

We first consider the case $\mu=0$. Then $f_{1}(t)=\mathrm{e}^{-\lambda t}$, and its $i$ th order derivative is simply $f_{1}^{(i)}(t)=(-\lambda)^{i} f_{1}(t)$. By the Euler-Maclaurin formula, for any integer $m \geq 1$, we have

$$
\begin{aligned}
E_{1} & =-\frac{1}{2 N}\left(\mathrm{e}^{-\lambda}-1\right)-\sum_{i=1}^{m} \frac{B_{2 i}}{(2 i) !} \frac{1}{N^{2 i}}\left(f_{1}^{(2 i-1)}(1)-f_{1}^{(2 i-1)}(0)\right)-R_{2 m+2} \\
& =\frac{1-\mathrm{e}^{-\lambda}}{2 N}-\sum_{i=1}^{m} \frac{B_{2 i}}{(2 i) !} \frac{1}{N^{2 i}} \lambda^{2 i-1}\left(1-\mathrm{e}^{-\lambda}\right)+O\left(\frac{1}{N^{2 m+2}}\right)
\end{aligned}
$$

where the last term is due to the boundedness of $\hat{B}_{2 m+2}$ and

$$
R_{2 m+2}=\frac{1}{N^{2 m+2}} \int_{0}^{1}\left(B_{2 m+2}-\hat{B}_{2 m+2}(N x)\right) \frac{f_{1}^{(2 m+2)}(x)}{(2 m+2) !} \mathrm{d} x
$$

Next, we consider the case when $\mu \neq 0$. In the following, we assume that $\mu>0$. When $\mu<0, \int_{0}^{\mu}$ is understood as $-\int_{\mu}^{0}$ and $x \in[0, \mu]$ is understood as $x \in[\mu, 0]$. Otherwise, the proof is identical. Note that $f_{1}(t)$ is not differentiable at 0 . Its derivatives contain the terms $1 / \sqrt{t}, 1 / t \sqrt{t}, \ldots$ The Euler-Maclaurin formula cannot be applied directly. Instead, we use (4) to obtain the following representation for the complementary error function term in $f_{1}(t)$ :

$$
\begin{aligned}
\operatorname{ErfC}\left(-\frac{\mu}{\sqrt{2 \sigma^{2}}} \sqrt{t}\right) & =-\frac{1}{\sqrt{2 \sigma^{2}}} \sqrt{t} \int_{0}^{\mu} \operatorname{ErfC}(1)\left(-\frac{x}{\sqrt{2 \sigma^{2}}} \sqrt{t}\right) \mathrm{d} x+\operatorname{ErfC}(0) \\
& =\sqrt{\frac{2}{\pi \sigma^{2}}} \sqrt{t} \int_{0}^{\mu} \exp \left(-\frac{x^{2}}{2 \sigma^{2}} t\right) \mathrm{d} x+1
\end{aligned}
$$


Similarly, we get the representation for $\operatorname{ErfC}\left(-\left(\mu / \sqrt{2 \sigma^{2}}\right) \sqrt{n / N}\right)$ by replacing $t$ with $n / N$ in the above. Thus, using our notation $a(x)=\lambda+x^{2} /\left(2 \sigma^{2}\right)>0$, we have

$$
\begin{aligned}
E_{1}= & \sqrt{\frac{2}{\pi \sigma^{2}}} \int_{0}^{\mu}\left(\int_{0}^{1} \sqrt{t} \mathrm{e}^{-a(x) t} \mathrm{~d} t-\frac{1}{N} \sum_{n=1}^{N} \sqrt{\frac{n}{N}} \mathrm{e}^{-a(x) n / N}\right) \mathrm{d} x \\
& +\left(\int_{0}^{1} \mathrm{e}^{-\lambda t} \mathrm{~d} t-\frac{1}{N} \sum_{n=1}^{N} \mathrm{e}^{-\lambda n / N}\right) .
\end{aligned}
$$

We have established the expansion for the second term above when discussing the case for $\mu=0$. To deal with the first term, we set

$$
\begin{aligned}
E_{1 x} & =\int_{0}^{1} g(t, x) \mathrm{d} t-\frac{1}{N} \sum_{n=1}^{N} g\left(\frac{n}{N}, x\right) \\
& =\left(\int_{0}^{\infty} g(t, x) \mathrm{d} t-\frac{1}{N} \sum_{n=1}^{\infty} g\left(\frac{n}{N}, x\right)\right)-\left(\int_{1}^{\infty} g(t, x) \mathrm{d} t-\frac{1}{N} \sum_{n=N+1}^{\infty} g\left(\frac{n}{N}, x\right)\right),
\end{aligned}
$$

where $g(t, x)=\sqrt{t} \mathrm{e}^{-a(x) t}$ was defined in (3c). By the definition of the Lerch transcendent, we have

$$
\frac{1}{N} \sum_{n=1}^{\infty} g\left(\frac{n}{N}, x\right)=\frac{\mathrm{e}^{-a(x) / N}}{N^{3 / 2}} \sum_{n=0}^{\infty}(n+1)^{1 / 2} \mathrm{e}^{-a(x) n / N}=\frac{\mathrm{e}^{-a(x) / N}}{N^{3 / 2}} \Phi\left(\mathrm{e}^{-a(x) / N},-\frac{1}{2}, 1\right) .
$$

For any positive integer $N$ such that

$$
N>\frac{2 \lambda \sigma^{2}+\mu^{2}}{4 \pi \sigma^{2}}
$$

we have $0<a(x) / N<2 \pi$ on $[0, \mu]$ and, hence, expansion (7) applies, i.e.

$$
\begin{aligned}
\frac{1}{N} \sum_{n=1}^{\infty} g\left(\frac{n}{N}, x\right) & =\Gamma\left(\frac{3}{2}\right)(a(x))^{-3 / 2}+\sum_{n=0}^{\infty} \zeta\left(-\frac{1}{2}-n\right) \frac{(-a(x))^{n}}{n !} \frac{1}{N^{n+3 / 2}} \\
& =\int_{0}^{\infty} g(t, x) \mathrm{d} t+\sum_{n=0}^{\infty} \zeta\left(-\frac{1}{2}-n\right) \frac{(-a(x))^{n}}{n !} \frac{1}{N^{n+3 / 2}},
\end{aligned}
$$

where we have used the fact that, for any $a>0, \int_{0}^{\infty} \sqrt{t} \mathrm{e}^{-a t} \mathrm{~d} t=\Gamma\left(\frac{3}{2}\right) / a^{3 / 2}$. We have thus obtained the expansion for the first part in $E_{1 x}$. For the second part, note that $g(t, x)$, as a function of $t$, is smooth on $[1, \infty)$, and all its derivatives with respective to $t$ vanish at $\infty$ and are absolutely integrable on $[1, \infty)$ due to the exponential tail. This can be seen from the following:

$$
g^{(i)}(t, x):=\frac{\partial^{i}}{\partial t^{i}} g(t, x)=\sum_{j=0}^{i}\left(\begin{array}{l}
i \\
j
\end{array}\right) \frac{\partial^{j}\left(\mathrm{e}^{-a(x) t}\right)}{\partial t^{j}} \frac{\partial^{i-j}(\sqrt{t})}{\partial t^{i-j}}, \quad i \geq 0 .
$$

For example, when $i=1$ and $i=2$, we have

$$
g^{(1)}(t, x)=\sqrt{t} \mathrm{e}^{-a(x) t}\left(\frac{1}{2 t}-a(x)\right), \quad g^{(2)}(t, x)=\sqrt{t} \mathrm{e}^{-a(x) t}\left(a(x)^{2}-\frac{a(x)}{t}-\frac{1}{4 t^{2}}\right) .
$$


From the Euler-Maclaurin formula,

$$
\int_{1}^{\infty} g(t, x) \mathrm{d} t-\frac{1}{N} \sum_{n=N+1}^{\infty} g\left(\frac{n}{N}, x\right)=\frac{\mathrm{e}^{-a(x)}}{2 N}+\sum_{i=1}^{m} \frac{B_{2 i}}{(2 i) !} \frac{1}{N^{2 i}} g^{(2 i-1)}(1, x)-R_{2 m+2}(x),
$$

where the remainder is given by

$$
R_{2 m+2}(x)=\frac{1}{N^{2 m+2}} \int_{1}^{\infty}\left(B_{2 m+2}-\hat{B}_{2 m+2}((t-1) N)\right) \frac{g^{(2 m+2)}(t, x)}{(2 m+2) !} \mathrm{d} t .
$$

Thus, we have the following expansion for $E_{1 x}$ :

$$
E_{1 x}=-\sum_{n=0}^{\infty} \zeta\left(-\frac{1}{2}-n\right) \frac{(-a(x))^{n}}{n !} \frac{1}{N^{n+3 / 2}}-\frac{\mathrm{e}^{-a(x)}}{2 N}-\sum_{i=1}^{m} \frac{B_{2 i}}{(2 i) !} \frac{1}{N^{2 i}} g^{(2 i-1)}(1, x)+R_{2 m+2}(x)
$$

Recall that $a(x)=\lambda+x^{2} /\left(2 \sigma^{2}\right)$. So we have

$$
\int_{0}^{\mu} \mathrm{e}^{-a(x)} \mathrm{d} x=\mathrm{e}^{-\lambda} \int_{0}^{\mu} \mathrm{e}^{-x^{2} /\left(2 \sigma^{2}\right)} \mathrm{d} x=\sqrt{\frac{\pi \sigma^{2}}{2}} \mathrm{e}^{-\lambda} \operatorname{Erf}\left(\frac{\mu}{\sqrt{2 \sigma^{2}}}\right) .
$$

Using $1+\operatorname{Erf}(x)=\operatorname{ErfC}(-x)$, we obtain the following expansion for $E_{1}$ (the validity of interchanging the summation and integration will be established shortly):

$$
\begin{aligned}
E_{1}= & \sqrt{\frac{2}{\pi \sigma^{2}}} \int_{0}^{\mu} E_{1 x} \mathrm{~d} x+\frac{1-\mathrm{e}^{-\lambda}}{2 N}-\sum_{i=1}^{m} \frac{B_{2 i}}{(2 i) !} \frac{1}{N^{2 i}} \lambda^{2 i-1}\left(1-\mathrm{e}^{-\lambda}\right)+O\left(\frac{1}{N^{2 m+1}}\right) \\
= & \frac{1-\mathrm{e}^{-\lambda} \operatorname{Erfc}\left(-\mu / \sqrt{2 \sigma^{2}}\right)}{2 N}-\sqrt{\frac{2}{\pi \sigma^{2}}} \sum_{n=0}^{\infty} \frac{\zeta(-1 / 2-n)}{n ! N^{n+3 / 2}} \int_{0}^{\mu}\left(-\lambda-\frac{x^{2}}{2 \sigma^{2}}\right)^{n} \mathrm{~d} x \\
& -\sum_{i=1}^{m} \frac{B_{2 i}}{(2 i) ! N^{2 i}}\left(\lambda^{2 i-1}\left(1-\mathrm{e}^{-\lambda}\right)+\sqrt{\frac{2}{\pi \sigma^{2}}} \int_{0}^{\mu} g^{(2 i-1)}(1, x) \mathrm{d} x\right)+O\left(\frac{1}{N^{2 m+2}}\right) .
\end{aligned}
$$

In the above, we have used the fact that

$$
\begin{aligned}
\left|\int_{0}^{\mu} R_{2 m+2} \mathrm{~d} x\right| & \leq \frac{1}{N^{2 m+2}} \int_{0}^{\mu} \int_{1}^{\infty}\left|B_{2 m+2}-\hat{B}_{2 m+2}((t-1) N)\right| \frac{\left|g^{(2 m+2)}(t, x)\right|}{(2 m+2) !} \mathrm{d} t \mathrm{~d} x \\
& =O\left(\frac{1}{N^{2 m+2}}\right),
\end{aligned}
$$

since, for a fixed $m, \hat{B}_{2 m+2}((t-1) N)$ is bounded by a constant independent of $N$ (see Section 1$)$, and, from (14), $\left|g^{(2 m+2)}(t, x)\right|$ is bounded by $\sqrt{t} \mathrm{e}^{-\lambda t}$ multiplied by a polynomial in $a(x)$ of finite order and the polynomial itself is bounded for $x \in[0, \mu]$. Finally, we need to validate the interchanging of the summation and integration:

$$
\int_{0}^{\mu} \sum_{n=0}^{\infty} \frac{\zeta(-1 / 2-n)}{n !}\left(\frac{-a(x)}{N}\right)^{n} \mathrm{~d} x=\sum_{n=0}^{\infty} \int_{0}^{\mu} \frac{\zeta(-1 / 2-n)}{n !}\left(\frac{-a(x)}{N}\right)^{n} .
$$


We use Equation (23.2.6) of [1] with $s=-\frac{1}{2}-n$ for the Riemann zeta function and Equation (6.1.12) of [1] for the gamma function to obtain, for any integer $n \geq 0$,

$$
\begin{aligned}
\frac{\zeta(-1 / 2-n)}{n !} & =-2(2 \pi)^{-n-3 / 2} \zeta\left(n+\frac{3}{2}\right) \sin \left(\frac{n \pi}{2}+\frac{\pi}{4}\right) \frac{\Gamma(n+3 / 2)}{n !} \\
& =-2(2 \pi)^{-n-3 / 2} \zeta\left(n+\frac{3}{2}\right) \sin \left(\frac{n \pi}{2}+\frac{\pi}{4}\right) \frac{(2 n+1) ! !}{2^{n+1} n !} \sqrt{\pi}
\end{aligned}
$$

where $(2 n+1) ! !=1 \cdot 3 \cdot 5 \cdots(2 n+1)$. But $|\sin (n \pi / 2+\pi / 4)|=\sqrt{2} / 2$,

$$
\frac{(2 n+1) ! !}{2^{n+1} n !}=(n+1) \frac{(2 n+1) ! !}{2^{n+1}(n+1) !}=(n+1) \frac{(2 n+1) ! !}{(2 n+2) ! !}<n+1,
$$

and

$$
\zeta\left(n+\frac{3}{2}\right) \leq \zeta\left(\frac{3}{2}\right)<\infty
$$

We thus have the following bound:

$$
\left|\frac{\zeta(-1 / 2-n)}{n !}\right| \leq \frac{n+1}{(2 \pi)^{n+1}} \zeta\left(\frac{3}{2}\right)
$$

Moreover, $0<a(x) / N \leq\left(\lambda+\mu^{2} /\left(2 \sigma^{2}\right)\right) / N$ for $x \in[0, \mu]$, and, by our condition (13), $0<\left(\lambda+\mu^{2} /\left(2 \sigma^{2}\right)\right) /(2 \pi \bar{N})<1$. It follows that

$$
\sum_{n=0}^{\infty} \frac{\zeta(-1 / 2-n)}{n !}\left(\frac{-a(x)}{N}\right)^{n}
$$

converges absolutely and uniformly. This validates the interchanging of the summation and integration.

\subsection{Analysis for $E_{2}$}

The second term $E_{2}$ in (12) concerns $f_{2}(t)$. Recall that $f_{2}(t)=\mathrm{e}^{-a t} / \sqrt{t}$ and $a=\lambda+$ $\mu^{2} / 2 \sigma^{2}$. Following the same approach used in [14] and Section 2.1, we have

$$
E_{2}=\left(\int_{0}^{\infty} f_{2}(t) \mathrm{d} t-\frac{1}{N} \sum_{n=1}^{\infty} f_{2}\left(\frac{n}{N}\right)\right)-\left(\int_{1}^{\infty} f_{2}(t) \mathrm{d} t-\frac{1}{N} \sum_{n=N+1}^{\infty} f_{2}\left(\frac{n}{N}\right)\right)
$$

For the first component above, with $N$ satisfying (13) and, hence, $a / N<2 \pi$, expansion (7) with $z=\mathrm{e}^{-a / N}, s=\frac{1}{2}$, and $v=1$ can be used. With $\Gamma\left(\frac{1}{2}\right)=\sqrt{\pi}, \zeta(s, 1)=\zeta(s)$, and $\int_{0}^{\infty} f_{2}(t) \mathrm{d} t=\sqrt{\pi / a}$, we obtain

$$
\frac{1}{N} \sum_{n=1}^{\infty} f_{2}\left(\frac{n}{N}\right)=\int_{0}^{\infty} f_{2}(t) \mathrm{d} t+\sum_{n=0}^{\infty}\left(\frac{\zeta(1 / 2-n)(-a)^{n}}{n !}\right) \frac{1}{N^{n+1 / 2}} .
$$

For the second component in $E_{2}$, we apply the Euler-Maclaurin formula for $f_{2}(t)$, which is smooth on $[1, \infty)$, with all its derivatives vanishing at $\infty$ and absolutely integrable on $[1, \infty)$ :

$$
\int_{1}^{\infty} f_{2}(t) \mathrm{d} t-\frac{1}{N} \sum_{n=N+1}^{\infty} f_{2}\left(\frac{n}{N}\right)=\frac{1}{2 N} f_{2}(1)+\sum_{i=1}^{m}\left(\frac{B_{2 i}}{(2 i) !} f_{2}^{(2 i-1)}(1)\right) \frac{1}{N^{2 i}}-R_{2 m+2} .
$$


The remainder is given by

$$
R_{2 m+2}=\frac{1}{N^{2 m+2}} \int_{1}^{\infty}\left(B_{2 m+2}-\hat{B}_{2 m+2}(N(t-1))\right) \frac{f_{2}^{(2 m+2)}(t)}{(2 m+2) !} \mathrm{d} t .
$$

Combining the above, we have the following expansion for $E_{2}$ :

$$
\begin{aligned}
E_{2}= & -\frac{\zeta(1 / 2)}{\sqrt{N}}-\frac{\mathrm{e}^{-\left(\lambda+\mu^{2} / 2 \sigma^{2}\right)}}{2 N}-\sum_{n=1}^{\infty} \frac{\zeta(1 / 2-n)(-a)^{n}}{n ! N^{n+1 / 2}}-\sum_{i=1}^{m} \frac{B_{2 i}}{(2 i) ! N^{2 i}} f_{2}^{(2 i-1)}(1) \\
& +O\left(\frac{1}{N^{2 m+2}}\right) .
\end{aligned}
$$

Remark 1. In the analyses of $E_{1}$ and $E_{2}$, due to the nondifferentiability of the corresponding integrands at the lower endpoints of the integrals, the Euler-Maclaurin formula cannot be used directly. This difficulty is overcome by using the expansion of the Lerch transcendent, which essentially gives us the expansion of the error of approximating an integral with the right endpoint rule. Moreover, the Lerch transcendent is only necessary in analyzing $E_{1}$ and $E_{2}$, which basically correspond to the term with $k=0$ in (1) (see also (9) and (11)). The applicability of the Lerch transcendent is therefore not restricted by the assumption of normal jump sizes.

\subsection{Analysis for $\boldsymbol{E}_{3}$}

The third term $E_{3}$ in (12) concerns the summations

$$
\sum_{k=1}^{\infty} \frac{\lambda^{k}}{k !} \int_{0}^{1} p_{k}(t) \mathrm{d} t, \quad \sum_{k=1}^{\infty} \frac{\lambda^{k}}{k !} \int_{0}^{1} q_{k}(t) \mathrm{d} t,
$$

where $p_{k}$ and $q_{k}$ are defined in (3d)-(3e). The derivatives of $p_{k}(t)$ and $q_{k}(t)$ can be computed as

$$
\begin{aligned}
q_{k}^{(1)}(t)= & p_{k}(t)+t p_{k}^{(1)}(t) \\
q_{k}^{(2)}(t)= & 2 p_{k}^{(1)}(t)+t p_{k}^{(2)}(t) \\
p_{k}^{(1)}(t)= & \mathbf{1}_{\{k \geq 2\}}(k-1) \mathrm{e}^{-\lambda t} t^{k-2} \operatorname{ErfC}\left(-\frac{\mu t+k \tilde{m}}{\sqrt{2\left(\sigma^{2} t+k s^{2}\right)}}\right) \\
& -\lambda p_{k}(t)+\mathrm{e}^{-\lambda t} t^{k-1} \phi\left(\frac{\mu t+k \tilde{m}}{\sqrt{\sigma^{2} t+k s^{2}}}\right) \frac{\mu \sigma^{2} t+2 \mu k s^{2}-k \sigma^{2} \tilde{m}}{\left(\sigma^{2} t+k s^{2}\right)^{3 / 2}}, \quad k \geq 1
\end{aligned}
$$

Recall that $\phi(x)$ is the probability density function of the standard normal distribution. We can further compute the derivative of $p_{k}^{(1)}(t)$. The derivative of the first term in (15) has a similar form as $p_{k}^{(1)}(t)$ itself. The derivatives of the remaining terms in (15) are also straightforward to compute. Using the boundedness of the normal density $\phi(x)$ and the complementary error function $\operatorname{Erfc}(x)$, it can be seen that, for any integer $i \geq 0, p_{k}^{(i)}(t)$ and $q_{k}^{(i)}(t)$ can all be bounded by polynomials in $k$ of finite orders on $[0,1]$. Consequently, we have

$$
\sum_{k=1}^{\infty} \frac{\lambda^{k}}{k !}\left|p_{k}^{(i)}(1)-p_{k}^{(i)}(0)\right|<\infty, \quad \sum_{k=1}^{\infty} \frac{\lambda^{k}}{k !} \int_{0}^{1}\left|p_{k}^{(i)}(t)\right| \mathrm{d} t<\infty,
$$


and

$$
\sum_{k=1}^{\infty} \frac{\lambda^{k}}{k !}\left|q_{k}^{(i)}(1)-q_{k}^{(i)}(0)\right|<\infty, \quad \sum_{k=1}^{\infty} \frac{\lambda^{k}}{k !} \int_{0}^{1}\left|q_{k}^{(i)}(t)\right| \mathrm{d} t<\infty .
$$

From the Euler-Maclaurin formula, using (17) and $q_{k}(0)=0, k \geq 1$, we obtain

$$
\begin{aligned}
\mu \sum_{k=1}^{\infty} & \frac{\lambda^{k}}{k !}\left(\int_{0}^{1} q_{k}(t) \mathrm{d} t-\frac{1}{N} \sum_{n=1}^{N} q_{k}\left(\frac{n}{N}\right)\right) \\
= & \mu \sum_{k=1}^{\infty} \frac{\lambda^{k}}{k !}\left(\frac{1}{2 N}\left(q_{k}(0)-q_{k}(1)\right)-\sum_{i=1}^{m} \frac{B_{2 i}}{(2 i) ! N^{2 i}}\left(q_{k}^{(2 i-1)}(1)-q_{k}^{(2 i-1)}(0)\right)\right) \\
& +O\left(\frac{1}{N^{2 m+2}}\right) \\
= & -\frac{\mu}{2 N} \sum_{k=1}^{\infty} \frac{\lambda^{k}}{k !} q_{k}(1)-\sum_{i=1}^{m} \frac{\mu B_{2 i}}{(2 i) ! N^{2 i}} \sum_{k=1}^{\infty} \frac{\lambda^{k}}{k !}\left(q_{k}^{(2 i-1)}(1)-q_{k}^{(2 i-1)}(0)\right)+O\left(\frac{1}{N^{2 m+2}}\right)
\end{aligned}
$$

Similarly, with $p_{k}(0)=0, k \geq 2$, from the Euler-Maclaurin formula and (16), we have

$$
\begin{aligned}
\tilde{m} \sum_{k=1}^{\infty} & \frac{\lambda^{k} k}{k !}\left(\int_{0}^{1} p_{k}(t) \mathrm{d} t-\frac{1}{N} \sum_{n=1}^{N} p_{k}\left(\frac{n}{N}\right)\right) \\
= & \frac{\tilde{m}}{2 N}\left(\lambda p_{1}(0)-\sum_{k=1}^{\infty} \frac{\lambda^{k} k}{k !} p_{k}(1)\right)-\sum_{i=1}^{m} \frac{\tilde{m} B_{2 i}}{(2 i) ! N^{2 i}} \sum_{k=1}^{\infty} \frac{\lambda^{k} k}{k !}\left(p_{k}^{(2 i-1)}(1)-p_{k}^{(2 i-1)}(0)\right) \\
& +O\left(\frac{1}{N^{2 m+2}}\right) .
\end{aligned}
$$

Adding the above, noting that $q_{k}(1)=p_{k}(1)$, we obtain the expansion for $E_{3}$ :

$$
\begin{aligned}
E_{3}= & \frac{1}{2 N}\left(\tilde{m} \lambda p_{1}(0)-\sum_{k=1}^{\infty} \frac{\lambda^{k}}{k !}(\mu+\tilde{m} k) p_{k}(1)\right) \\
& -\sum_{i=1}^{m} \frac{B_{2 i}}{(2 i) ! N^{2 i}} \sum_{k=1}^{\infty} \frac{\lambda^{k}}{k !}\left(\mu\left(q_{k}^{(2 i-1)}(1)-q_{k}^{(2 i-1)}(0)\right)+\tilde{m} k\left(p_{k}^{(2 i-1)}(1)-p_{k}^{(2 i-1)}(0)\right)\right) \\
& +O\left(\frac{1}{N^{2 m+2}}\right) .
\end{aligned}
$$

\subsection{Analysis for $\boldsymbol{E}_{4}$}

The fourth term $E_{4}$ in (12) concerns the summation

$$
\sum_{k=1}^{\infty} \frac{\lambda^{k}}{k !} \int_{0}^{1} r_{k}(t) \mathrm{d} t
$$

where $r_{k}$ is defined in (3f). The first-order derivative of $r_{k}(t)$ is given by

$$
\begin{aligned}
r_{k}^{(1)}(t)= & \mathbf{1}_{\{k \geq 2\}}(k-1) \mathrm{e}^{-\lambda t} t^{k-2} \sqrt{\sigma^{2} t+k s^{2}} \exp \left(-\frac{(\mu t+k \tilde{m})^{2}}{2\left(\sigma^{2} t+k s^{2}\right)}\right) \\
& +r_{k}(t)\left(-\lambda+\frac{\sigma^{2}}{2\left(\sigma^{2} t+k s^{2}\right)}-\frac{(\mu t+k \tilde{m})\left(\mu \sigma^{2} t+2 \mu k s^{2}-k \tilde{m} \sigma^{2}\right)}{2\left(\sigma^{2} t+k s^{2}\right)^{2}}\right) \quad \text { for } k \geq 1 .
\end{aligned}
$$


We can easily compute higher-order derivatives of $r_{k}(t)$. For example, the derivative of the first term in $r_{k}^{(1)}(t)$ has a similar form as $r_{k}^{(1)}(t)$ itself. The derivatives of the remaining terms, although cumbersome, are straightforward to compute. We observe that $r_{k}(t)$ is in fact smooth on $[0,1]$, and, for any integer $i \geq 0, r_{k}^{(i)}(t)$ can be bounded by a polynomial in $k$ of finite orders on $[0,1]$. Consequently, we have

$$
\sum_{k=1}^{\infty} \frac{\lambda^{k}}{k !}\left|r_{k}^{(i)}(1)-r_{k}^{(i)}(0)\right|<\infty, \quad \sum_{k=1}^{\infty} \frac{\lambda^{k}}{k !} \int_{0}^{1}\left|r_{k}^{(i)}(t)\right| \mathrm{d} t<\infty .
$$

Therefore, by the Euler-Maclaurin formula, using $r_{k}(0)=0$ for $k \geq 2$, we obtain the following expansion for $E_{4}$ :

$$
\begin{aligned}
E_{4}= & \sum_{k=1}^{\infty} \frac{\lambda^{k}}{k !}\left(\int_{0}^{1} r_{k}(t) \mathrm{d} t-\frac{1}{N} \sum_{n=1}^{N} r_{k}\left(\frac{n}{N}\right)\right) \\
= & \sum_{k=1}^{\infty} \frac{\lambda^{k}}{k !}\left(\frac{1}{2 N}\left(r_{k}(0)-r_{k}(1)\right)-\sum_{i=1}^{m} \frac{B_{2 i}}{(2 i) ! N^{2 i}}\left(r_{k}^{(2 i-1)}(1)-r_{k}^{(2 i-1)}(0)\right)\right)+O\left(\frac{1}{N^{2 m+2}}\right) \\
= & \frac{1}{2 N}\left(\lambda r_{1}(0)-\sum_{k=1}^{\infty} \frac{\lambda^{k}}{k !} r_{k}(1)\right)-\sum_{i=1}^{m} \frac{B_{2 i}}{(2 i) ! N^{2 i}} \sum_{k=1}^{\infty} \frac{\lambda^{k}}{k !}\left(r_{k}^{(2 i-1)}(1)-r_{k}^{(2 i-1)}(0)\right) \\
& +O\left(\frac{1}{N^{2 m+2}}\right) .
\end{aligned}
$$

Combining the above results for $E_{1}, E_{2}, E_{3}$, and $E_{4}$, we obtain the expansion in Theorem 2 .

\section{Concluding remarks}

We studied the monitoring error of the supremum of Merton's normal jump diffusion process on a fixed finite time horizon. The error is of the form $a_{0} / N^{1 / 2}+a_{1} / N^{3 / 2}+\cdots+b_{1} / N+b_{2} / N^{2}+$ $b_{4} / N^{4}+\cdots$. The coefficients $\left\{a_{0}, a_{1}, \ldots, b_{1}, b_{2}, \ldots\right\}$ admit explicit expressions and can be computed numerically. The proof used Spitzer's identity regarding the maximum of a random walk and the Euler-Maclaurin formula regarding the discretization error of the trapezoidal sum approximation to an integral, as well as a well-known expansion for the Lerch transcendent. The Lerch transcendent has been successfully applied in [13] and [14] to study the maximum of a Gaussian random walk and the monitoring error of the supremum of a drifted Brownian motion. The same technique was used in deriving our main result, Theorem 2. Finally, we remark that the analysis in this paper relies on the explicit expression of the transition probability density of the underlying stochastic process. For other jump diffusion processes and more general Lévy processes, the transition densities may not be available explicitly or may be too complex. In these cases, expansions of the monitoring errors can be obtained by analyzing the characteristic functions or Lévy densities of the underlying processes. The extension to more general Lévy processes will be reported separately.

\section{References}

[1] Abramowitz, M. and Stegun, I. A. (1964). Handbook of Mathematical Functions with Formulas, Graphs, and Mathematical Tables. US Government Printing Office, Washington, DC.

[2] Asmussen, S., Glynn, P. and Pitman, J. (1995). Discretization error in simulation of one-dimensional reflecting Brownian motion. Ann. Appl. Prob. 5, 875-896. 
[3] Black, F. and Scholes, M. (1973). The pricing of options and corporate liabilities. J. Political Econom. 81, 637-654.

[4] Boyarchenko, S. I. And Levendorskiř, S. Z. (2002). Non-Gaussian Merton-Black-Scholes Theory. World Scientific, River Edge, NJ.

[5] Broadie, M., Glasserman, P. and Kou, S. G. (1999). Connecting discrete and continuous path-dependent options. Finance Stoch. 3, 55-82.

[6] Calvin, J. M. (1995). Average performance of passive algorithms for global optimization. J. Math. Anal. Appl. 191, 608-617.

[7] Cont, R. and Tankov, P. (2004). Financial Modelling with Jump Processes. Chapman \& Hall/CRC, Boca Raton, FL.

[8] DAhlQuist, G. AND BJöRCK, Å. (2008). Numerical Methods in Scientific Computing, Vol. I. Society for Industrial and Applied Mathemetics, Philadelphia, PA.

[9] Erdelyi, A., Magnus, W., Oberhettinger, F. and Tricomi, F. (1953). Higher Transcendental Functions, Vol. I. McGraw Hill, New York.

[10] Feng, L. And Linetsky, V. (2008). Pricing discretely monitored barrier options and defaultable bonds in Lévy process models: a fast Hilbert transform approach. Math. Finance 18, 337-384.

[11] Feng, L. And Linetsky, V. (2009). Computing exponential moments of the discrete maximum of a Lévy process and lookback options. Finance Stoch. 13, 501-529.

[12] Gould, H. W. (1972). Explicit formulas for Bernoulli numbers. Amer. Math. Monthly 79, 44-51.

[13] Janssen, A. J. E. M. and van Leeuwatrden, J. S. H. (2007). On Lerch's transcendent and the Gaussian random walk. Ann. Appl. Prob. 17, 421-439.

[14] Janssen, A. J. E. M. and van Leeuwaarden, J. S. H. (2009). Equidistant sampling for the maximum of a Brownian motion with drift on a finite horizon. Electron. Commun. Prob. 14, 143-150.

[15] Jeannin, M. And Pistorius, M. (2010). A transform approach to compute prices and greeks of barrier options driven by a class of Lévy processes. Quant. Finance 10, 629-644.

[16] Kou, S. G. (2008). Discrete barrier and lookback options. In Handbooks in Operations Research and Management Science, Vol. 15, eds J. Birge and V. Linetsky, pp. 343-373.

[17] Kou, S. G. AND WANG, H. (2004). Option pricing under a double exponential jump diffusion model. Manag. Sci. 50, 1178-1192.

[18] Kudryavtsev, O. And LevendorskiĬ, S. (2009). Fast and accurate pricing of barrier options under Lévy processes. Finance Stoch. 13, 531-562.

[19] Kuznetsov, A., Kyprianou, A., Pardo, J. and van Schaik, K. (2010). A Wiener-Hopf Monte-Carlo simulation technique for Lévy processes. Working paper.

[20] Merton, R. C. (1973). Theory of rational option pricing. Bell J. Econom. Manag. Sci. 4, 141-183.

[21] Merton, R. C. (1976). Option pricing when underlying stock returns are discontinuous. J. Financial Econom. 3, 125-144.

[22] Petrella, G. And Kou, S. (2004). Numerical pricing of discrete barrier and lookback options via Laplace transforms. J. Comput. Finance 8, 1-37.

[23] Schoutens, W. (2003). Lévy Processes in Finance: Pricing Financial Derivatives. John Wiley, Hoboken, NJ.

[24] Spitzer, F. (1956). A combinatorial lemma and its application to probability theory. Trans. Amer. Math. Soc. 82, 323-339. 